\title{
Effect of Intracavitary Probe Size on the Accuracy of Inverse Endocardial Potential Estimates
}

\author{
EN Bender ${ }^{1}$, LG Olson $^{1}$, RD Throne ${ }^{1}$, JR Windle ${ }^{2}$ \\ ${ }^{1}$ Rose-Hulman Institute of Technology, Terre Haute, IN, USA \\ ${ }^{2}$ University of Nebraska Medical Center, Omaha, NE, USA
}

\begin{abstract}
We simulated the effects of probe size on the accuracy of an intracavitary probe. Measured data was used to find the potentials over the entire endocardial surface. These "original" endocardial potentials were used with two models, one with a standard probe and the other with a probe half as large, to compute corresponding probe potentials. Random Gaussian noise was added to these "zero-noise" probe potentials. Using first-order Tikhonov regularization, the endocardial potentials for both models were estimated. Making the probe smaller had very little effect on the CC between the original endocardial signals and the estimated endocardial signals. These results suggest that even a probe half as large as that currently used clinically could be effectively used for patient treatment, so long as higher order regularization methods are employed.
\end{abstract}

\section{Introduction}

Intracavitary probes which produce real-time inverse electrocardiographic estimates of endocardial potentials are currently in successful clinical use. However, in some instances the probe is too large for the cardiac chamber. Because it is not clear how reducing the size of the probe might change its accuracy, this study was undertaken to simulate the accuracy of a normal probe compared to a smaller probe- one half as large in each dimension as the original probe.

\section{Methods}

An Ensite $3000^{T M}$ noncontact probe (Endocardial Solutions Inc.) was inserted into the left ventricle of a patient undergoing endocardial mapping. The Ensite system measures the location of the probe and the ablation catheter as the catheter is moved. The Ensite system also records geometric information for the surface of the ventricle, and creates a computer model of the volume.
At fifteen different locations in the left ventricle a sinus rhythm depolarization was recorded. These asynchronous recordings were then synchronized in time by aligning body surface ECG signals.

A finite element model of the volume between the probe surface and the endocardial surface was generated using the original volume model created by the Ensite system. Figure 1 shows the finite element mesh, which will be called the "normal-size" model. Next, the dimensions of the probe surface were all reduced by a factor of 2 , and a new finite element model of the volume was created. Figure 2 shows this "half-size" model. From these models, transfer matrices relating the endocardial and probe potentials were created using standard techniques.

We employed four inverse algorithms in this study. Zero-order Tikhonov regularization is a widely-used and very robust algorithm [1, 2]. First-order Tikhonov regularization is also a standard algorithm, but is less widely used, and the regularization matrix must be implemented carefully in order to obtain good results [3]. The third and fourth methods employed are the zeroand first-order Generalized Eigensystem method [4] which often show improved performance but are less widely used.

In order to examine the effect of probe size, potential values on the entire endocardial surface (not just the 15 measured sites) were required. For the original probe, these endocardial potentials were estimated from the measured probe data with all four inverse methods. Since each method incorporates an adjustable parameter which controls the relative importance of either amplitude or smoothness (regularization) and fidelity to the measured data, this adjustable parameter was selected as the single value over the entire depolarization which minimized the relative error and correlation coefficient between the estimates and the 15 measured endocardial signals. Next, these four estimates were averaged, and the resulting data set is referred to as the "original" endocardial data, $\phi_{E}$.

After the original endocardial data is created, it can be used to generate corresponding sets of probe data for the normal and small probes. Multiplying the endocardial 
Table 1. Normal-size probe: inverse solution errors for first-order Tikhonov regularization

\begin{tabular}{|ccc|}
\hline Noise Level (\%) & CC (mean \pm std) & RE (mean \pm std) \\
\hline 1 & $0.99 \pm 0.0003$ & $0.12 \pm 0.0016$ \\
2 & $0.99 \pm 0.0004$ & $0.15 \pm 0.0020$ \\
5 & $0.98 \pm 0.0016$ & $0.20 \pm 0.0050$ \\
10 & $0.96 \pm 0.0047$ & $0.27 \pm 0.0108$ \\
20 & $0.93 \pm 0.0060$ & $0.42 \pm 0.0168$ \\
\hline
\end{tabular}

Table 2. Half-size probe: inverse solution errors for firstorder Tikhonov regularization

\begin{tabular}{|ccc|}
\hline Noise Level (\%) & CC (mean \pm std $)$ & RE $($ mean \pm std $)$ \\
\hline 1 & $0.98 \pm 0.0008$ & $0.20 \pm 0.0025$ \\
2 & $0.97 \pm 0.0011$ & $0.23 \pm 0.0039$ \\
5 & $0.96 \pm 0.0018$ & $0.30 \pm 0.0046$ \\
10 & $0.95 \pm 0.0019$ & $0.37 \pm 0.0066$ \\
20 & $0.93 \pm 0.0032$ & $0.48 \pm 0.0101$ \\
\hline
\end{tabular}

data, $\phi_{E}$, by the appropriate transfer matrix $\mathbf{Z}_{E P}^{H}$ (for the half-size probe) or $\mathbf{Z}_{E P}^{N}$ (for the normal-size probe) gives the sets of data on the probe surface.

Random Gaussian noise was added to the probe data for both the half-size probe $\left(\phi_{P}^{H}\right)$ and the normal-size probe $\left(\phi_{P}^{N}\right)$. The noise had a mean value of zero and a standard deviation $\sigma=F A_{R M S}$, where $A_{R M S}$ is the root-meansquare amplitude of the probe potentials and $\mathrm{F}$ is noise level used. $\mathrm{F}=0.01,0.02,0.05,0.10$, and 0.20 correspond to noise levels of $1 \%$ through $20 \%$.

First-order Tikhonov regularization was then used to create endocardial potential estimates from the noisy probe data, both for the normal-size probe and the half-size probe. The Combined Residual Error and Smoothing Operator (CRESO) technique [5] was used to choose the regularization parameter at each time instant, and 25 simulations were run at each noise level. For each estimate, the correlation coefficient (CC) between the "original" endocardial data and estimated potentials was computed, as well as the relative error (RE) between the two signals.

\section{Results}

Table 1 shows the inverse solution errors for the normalsize probe with first-order Tikhonov regularization, while Table 2 shows the inverse errors for the half-size probe.

Figure 3 shows the norm of the endocardial potential over the depolarization for reference. Figures 4, 5, and 6 show the original endocardial potentials at $70 \mathrm{~ms}$ as well as the endocardial potentials estimated with the normalsize probe and the half-size probe. Figures 7 through 12 show similar plots for $90 \mathrm{~ms}$ and $110 \mathrm{~ms}$.

\section{Discussion and conclusions}

Making the probe smaller had very little effect on the CC between the original endocardial signals and the estimated endocardial signals. With $5 \%$ added noise the CC for the normal probe was 0.98 , compared to 0.96 on the small probe. The effect on the RE was more substantial: at a $5 \%$ noise level the RE was 0.2 for the normal probe and 0.3 for the small probe. These results suggest that even a probe half as large as that currently used clinically could be effectively used for patient treatment, so long as higher order regularization methods are employed.

\section{Acknowledgements}

We would like to thank ESI and its Applied Research group for providing access to the data. This work was supported in part by the National Science Foundation grant BES-0240910.

\section{References}

[1] Rudy Y, Messinger-Rapport BJ. The inverse problem in electrocardiography: Solutions in terms of epicardial potentials. CRC Critical Review in Biomedical Engineering 1988;16:215-268.

[2] Johnston PR, Gulrajani R. A new method for regularization parameter determination in the inverse problem of electrocardiography. IEEE Transactions on Biomedical Engineering 1997;44:19-39.

[3] Throne RD, Olson LG, Hrabik TJ. A comparison of higher-order generalized eigensystem techniques and Tikhonov regularization for the inverse problem of electrocardiography. Inverse Problems in Engineering 1999; 7:143-193.

[4] Throne RD, Olson LG. Fusion of body surface potential and body surface Laplacian signals for electrocardiographic imaging. IEEE Transactions on biomedical engineering 2000;47:452-462.

[5] Colli-Franzone P, Guerri L, Taccardi B, Viganotti C. Finite element approximation of regularized solutions of the inverse potential problem of electrocardiography and applications to experimental data. Calcolo 1985;22:91-186.

Address for correspondence:

Lorraine G. Olson

Department of Mechanical Engineering

CM164

Rose-Hulman Institute of Technology

Terre Haute, IN 47803

Lorraine.Olson@Rose-Hulman.edu 


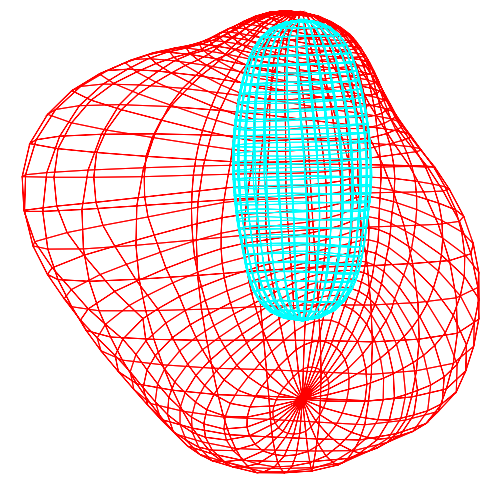

Figure 1. "Normal-size" finite element mesh for the patient used in this study. (5 element layers.)

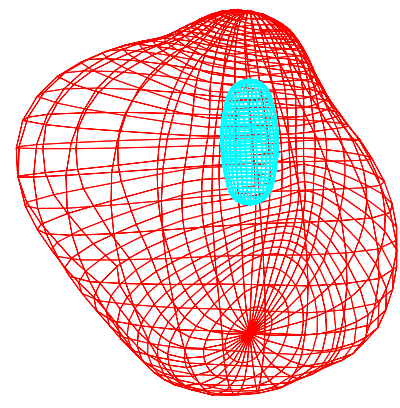

Figure 2. "Half-size" finite element mesh for the patient used in this study. (5 element layers.)

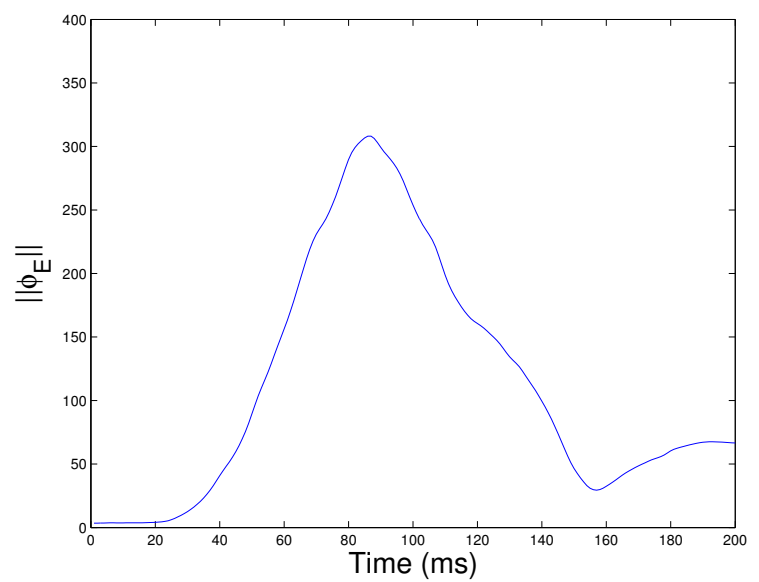

Figure 3. Norm of the endocardial potentials over time.

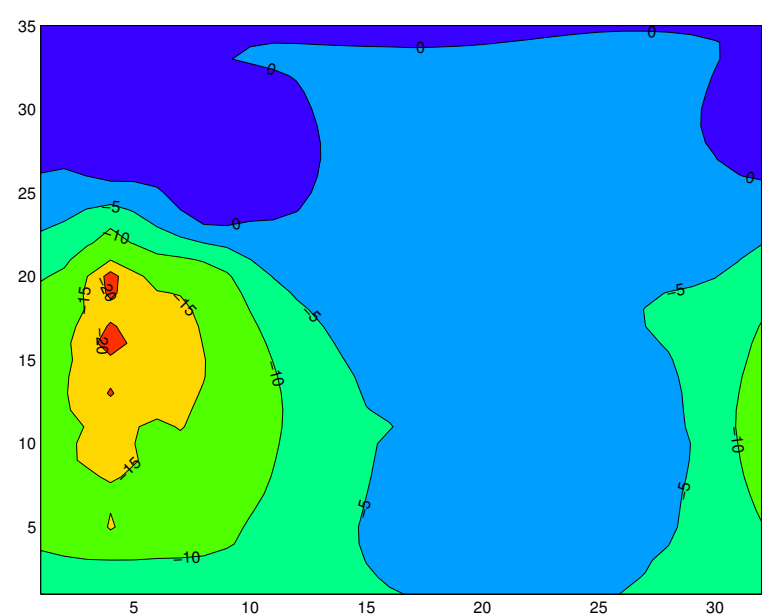

Figure 4. Original endocardial potentials at $70 \mathrm{~ms}$.

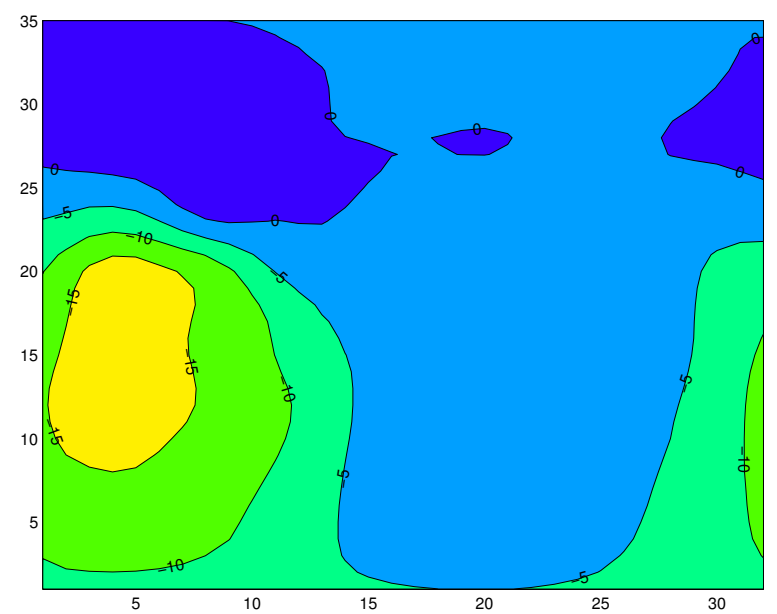

Figure 5. Estimated endocardial potentials using normalsize probe at $70 \mathrm{~ms}$.

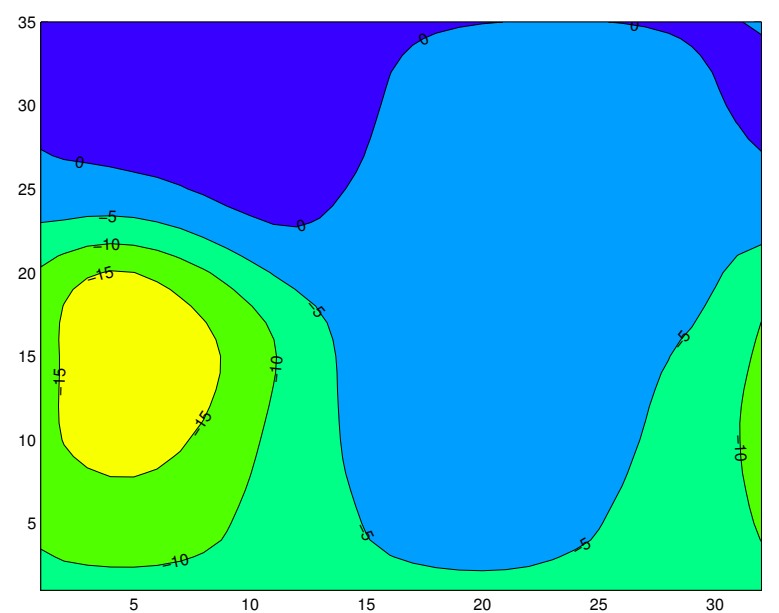

Figure 6. Estimated endocardial potentials using half-size probe at $70 \mathrm{~ms}$. 


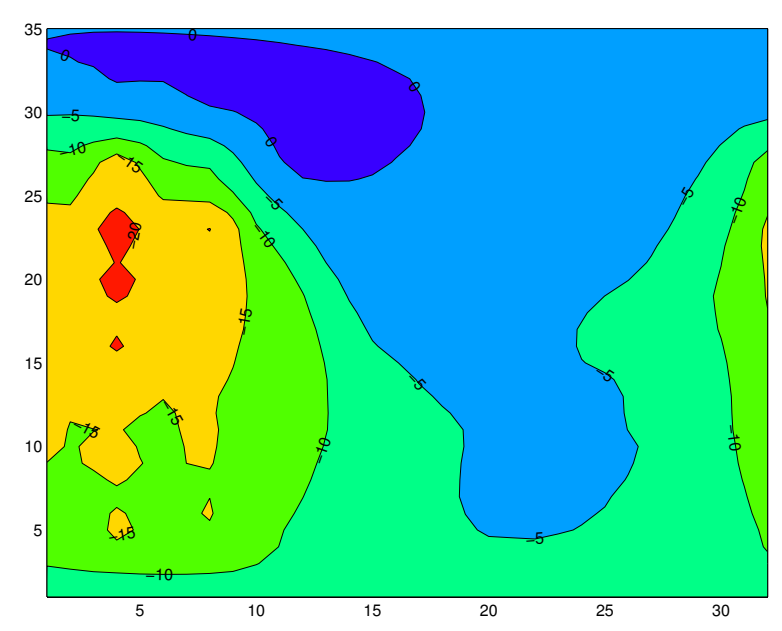

Figure 7. Original endocardial potentials at $90 \mathrm{~ms}$.

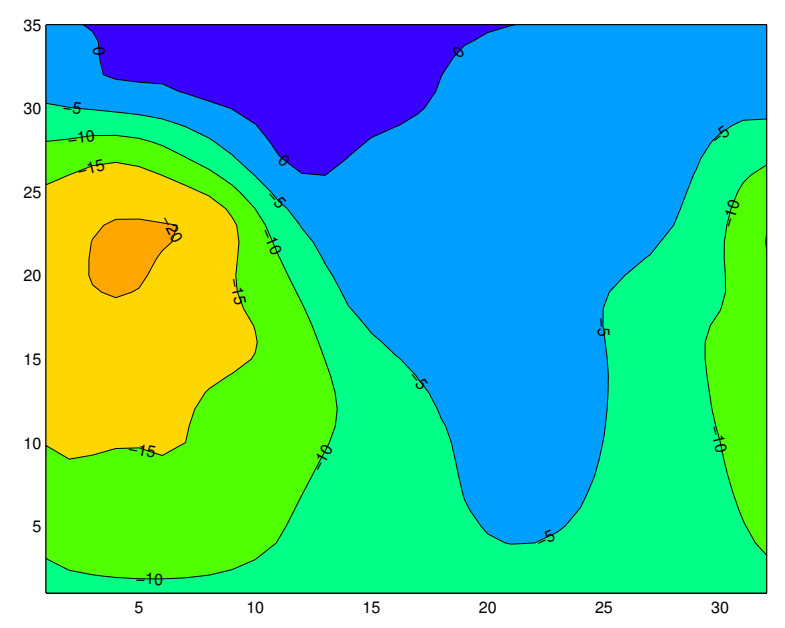

Figure 8. Estimated endocardial potentials using normalsize probe at $90 \mathrm{~ms}$.

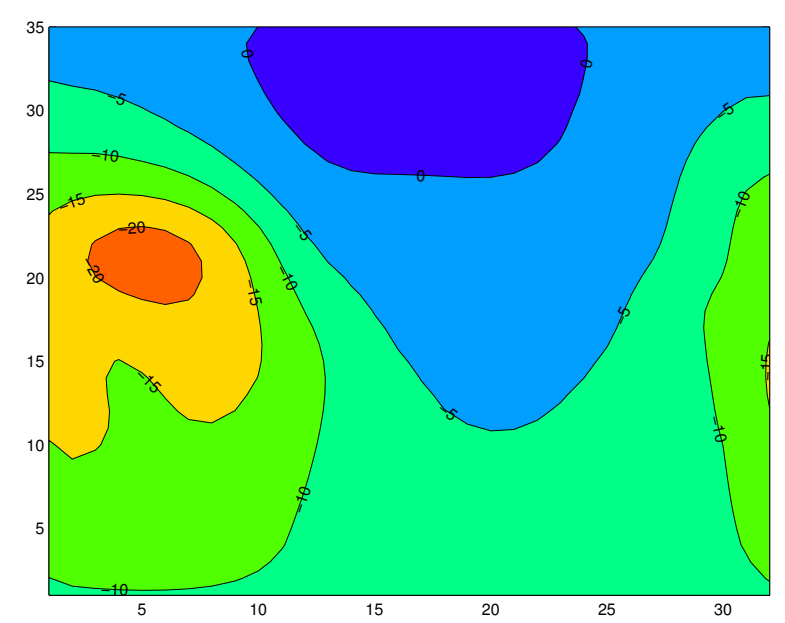

Figure 9. Estimated endocardial potentials using half-size probe at $90 \mathrm{~ms}$.

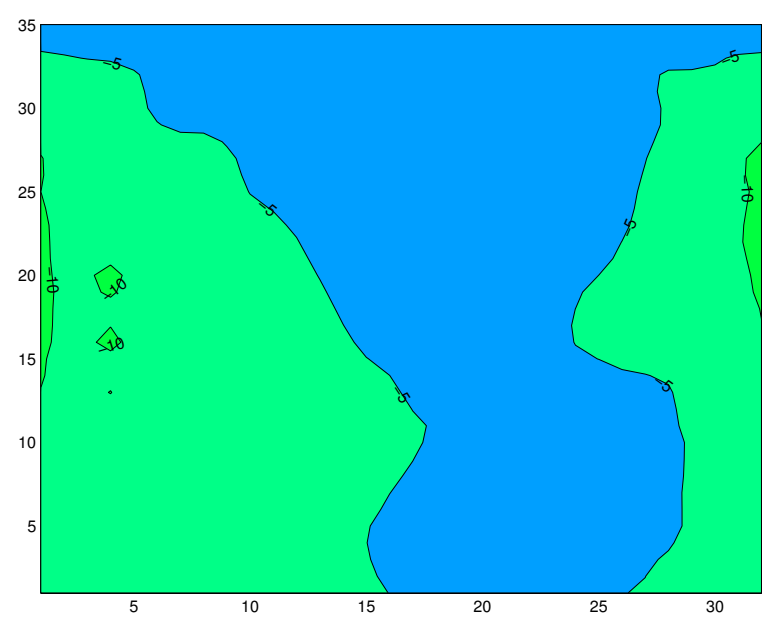

Figure 10. Original endocardial potentials at $110 \mathrm{~ms}$.

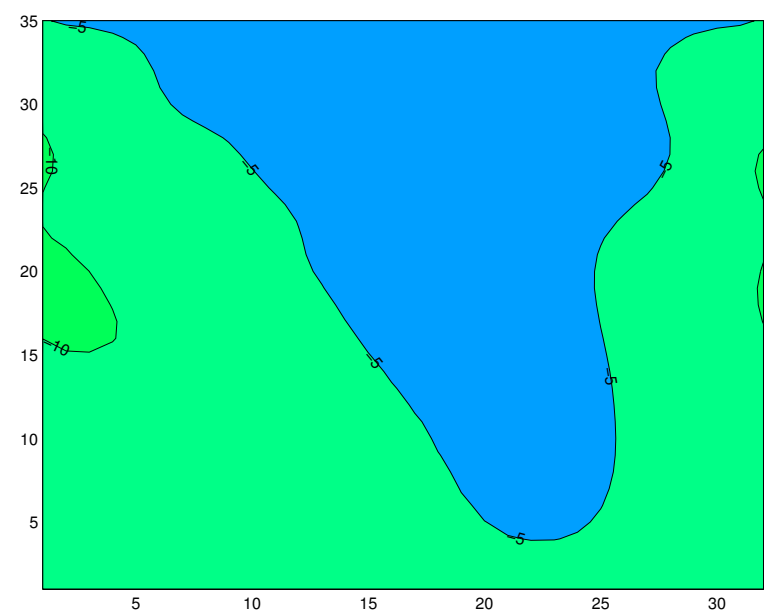

Figure 11. Estimated endocardial potentials using normalsize probe at $110 \mathrm{~ms}$.

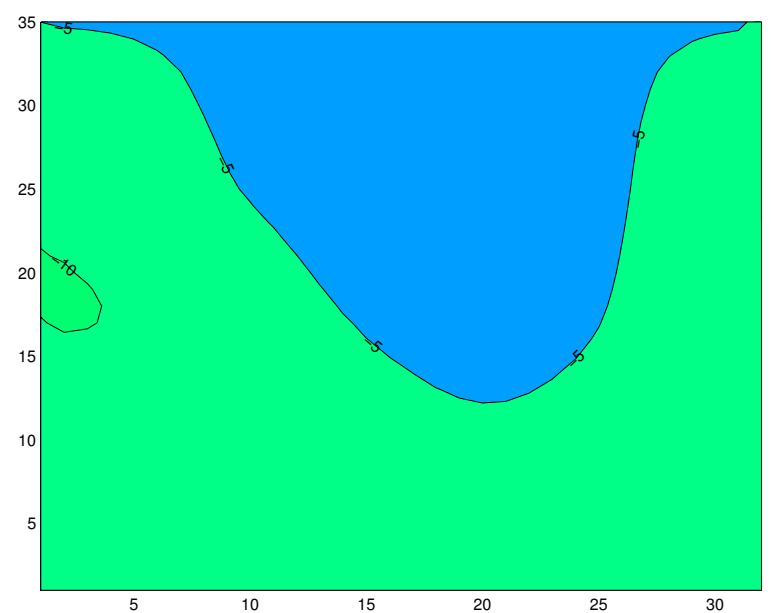

Figure 12. Estimated endocardial potentials using halfsize probe at $110 \mathrm{~ms}$. 